\title{
MEASUREMENTS OF SOLAR MAGNETIC FIELDS USING RADIO OBSERVATIONS WITH THE RATAN-600
}

\section{G. B. GELFREIKH, V. M. BOGOD}

Special Astrophysical Observatory, Russian Academy of Sciences, St. Petersburg 196140, Russia

\author{
V. E. ABRAMOV-MAXIMOV, S. V. TSVETKOV \\ Pulkovo Astronomical Observatory, Russian Academy of Sciences, \\ St. Petersburg 196140, Russia
}

\begin{abstract}
Three ways of measuring magnetic fields in the corona and chromosphere have been developed based on spectral polarization observations with high spatial resolution using the radio telescope RATAN600 . The methods rely on effects from the theory of the generation and propagation of microwaves in the solar atmosphere: thermal bremsstrahlung, thermal cyclotron emission, and inversion of the polarization sign in quasi-transverse field regions. The new radio spectrograph (PAS) at the RATAN-600 resulted in higher accuracy measurements of the strength and structure of coronal magnetic fields. Using observations made with the PAS on 1992 January 10, we show that the magnetic field strength in the lower corona above all large sunspots can be measured with an accuracy $\approx 3 \%$. The correlation with photospheric magnetic fields is good, 'radio' magnetic fields being weaker by $\approx 20 \%$.
\end{abstract}

\section{INTRODUCTION}

Several ways of making unique measurements of coronal and chromospheric magnetic fields are provided by radio observations based on mechanisms of generation of the radio emission and its propagation in anisotropic plasma. All the methods need both high spatial and spectral resolution, and accurate polarization measurements. The radio telescope RATAN-600 has good wavelength coverage: from 0.8 to $30 \mathrm{~cm}$ with detailed analysis of the spectra when using the new Panoramic Analyzer of the Spectrum (PAS) (Bogod et al., these proceedings). This new technique produces a measurement accuracy of $\sim 3 \%$ for magnetic fields of sunspots at the base of the solar corona. The spatial resolution of the instrument is $\sim 15$ arc sec at $\lambda=1.7 \mathrm{~cm}$, but only in one direction. The developed methods are:

(i) Thermal bremsstrahlung in a magnetic field generates polarized emission with an excess of the extraordinary mode. For weak magnetic fields a solution of the transfer equations (Gelfreikh 1972; Bogod et al. 1980) results in the 
following formula:

$$
B_{l}=\frac{107 \cdot P \%}{n \lambda},
$$

where $B_{l}$ is the longitudinal component of $B$, and polarization percentage, $P \%$, and spectral index, $n$, are found from observations at wavelength $\lambda$.

(ii) Measurements of emission spectra generated by the thermal cyclotron mechanism at the second and third harmonics of the gyrofrequency of electrons (Zheleznyakov 1970). The magnetic field strength $B$ is given by

$$
B=\frac{5400}{\lambda}(s=2) \& B=\frac{3570}{\lambda}(s=3),
$$

where $s$ is the harmonic number. We may often distinguish the second and third harmonics using polarization measurements (the third harmonic is strongly circularly polarized). Spectral observations allow us to find the shortest wavelength generated by this mechanism, corresponding to the strongest magnetic field in the corona. This method has been used to measure the magnetic fields above sunspots in the corona (Akhmedov et al. 1982, 1983; Abramov-Maximov \& Gelfreikh 1983, Bogod et al. 1982, Gelfreikh et al. 1985, Krüger et al. 1988). The improved accuracy of such measurements afforded by the PAS are illustrated below.

(iii) Analysis of inversion of the sign of circular polarization as a function of wavelength and time (Gelfreikh et al. 1987). This is due to propagation of an electromagnetic wave in a quasi-transverse magnetic field (Zheleznyakov 1970). The theory gives the expression for $B_{t}$ in QT-region:

$$
B_{t}=\frac{1.57 \cdot 10^{8}}{\lambda\left(\lambda N_{e} L_{B}\right)^{1 / 3}}
$$

where $N_{e}$ is the electron density, and $L_{B}$ is the scale length of the magnetic field. We identify the wavelength of the inversion of polarization, $\lambda_{i}$, with $\lambda$ in the formula above (Bogod et al., these proceedings).

\section{'RADIO' MAGNETIC FIELDS OF SUNSPOTS}

We illustrate the measurement of coronal magnetic fields above sunspots using PAS observations with the RATAN-600. Figure 1 shows scans of the solar disk in circular polarization at eight frequencies in the wavelength range of 1.8 to $3.2 \mathrm{~cm}$. On the upper curve one can identify up to 13 sources connected with sunspots. Only one $(\mathrm{g})$ is strong at the shortest wavelength $\lambda=1.8 \mathrm{~cm}$, implying that its coronal magnetic field exceeds $2000 \mathrm{G}$. For the rest of the sources polarized emission begins at longer wavelengths within the observed range. The sign of polarization is expected to correspond to an excess of an extraordinary mode.

Measurements of sunspot magnetic fields associated with the sources above are presented in Table I. In this table, $\mathrm{LS}=$ local source as it is marked in Figure 1, No.SG $=$ number of the corresponding sunspot group according to Solnechnye Dannye, $B_{\text {opt }}$ and $B_{\text {rad }}=$ magnetic field strengths found from optical and radio observations, $\lambda_{c r i t}=$ shortest wavelength range of emission, and $\eta=B_{\text {rad }} / B_{\text {opt }}$ (the mean value of $\eta=0.78$ ). 


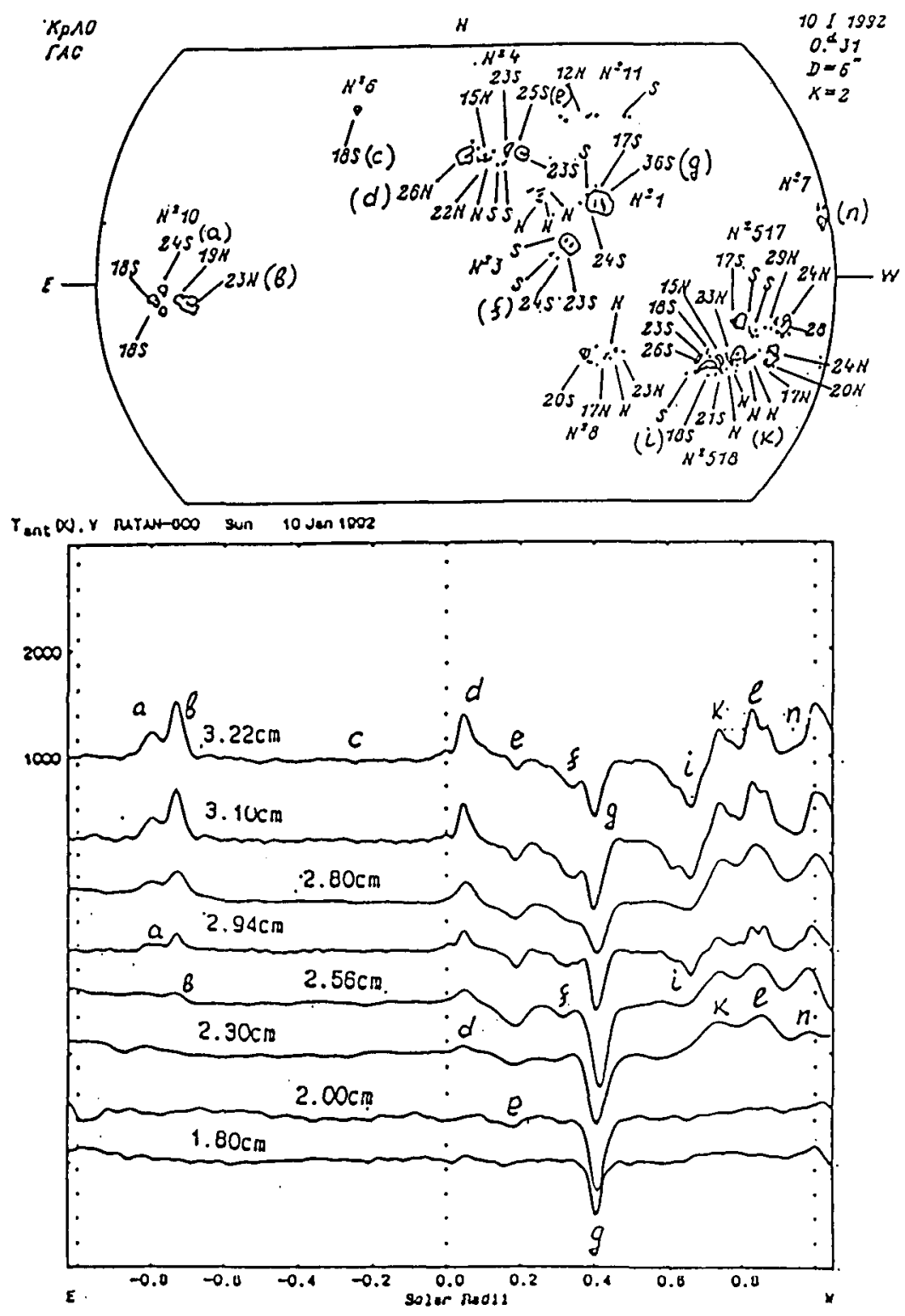

Fig. 1. Solar scans of circularly polarized radio emission made with the PAS on the RATAN-600 on 1992 January 10. An optical map of sunspots and their magnetic fields is also presented from the bulletin Solnechnye Dannye. 
TABLE I Magnetic fields of sunspots on 1992 January 10

\begin{tabular}{|c|c|c|c|c|c|c|}
\hline$\overline{\mathrm{LS}}$ & No.SG & $\overline{B_{\text {opt }}}$ & $B_{\text {rad }}$ & $\overline{\lambda_{\text {crit }}}$ & $\eta$ & Comments \\
\hline a & 10 & $24 \mathrm{~S}$ & 13.2 & $2.84 /(2.56) \mathrm{R}$ & $(0.55)$ & inver. of polar. \\
\hline b & 10 & $21 N$ & 16.6 & $2.30 /(2.00) \mathrm{R}$ & 0.79 & \\
\hline c & 6 & $18 \mathrm{~S}$ & 11.3 & $3.22 /(3.10) \mathrm{L}$ & 0.63 & \\
\hline d & 4 & $26 \mathrm{~N}$ & 16.6 & $2.30 /(2.00) \mathrm{R}$ & 0.64 & \\
\hline e & 4 & $25 \mathrm{~S}$ & 18.9 & $2.00 /(1.80) \mathrm{L}$ & 0.76 & \\
\hline$f$ & 3 & $23 \mathrm{~S}$ & 14.7 & $2.56 /(2.30) \mathrm{L}$ & 0.64 & \\
\hline g & 1 & $36 \mathrm{~S}$ & $>20$ & $\ll 1.80 \mathrm{~L}$ & $>0.56$ & \\
\hline $\mathrm{h}$ & 518 & $21 N$ & 16.6 & $2.30 /(2.00) R$ & 0.79 & \\
\hline $\mathrm{i}$ & 518 & $26 \mathrm{~S}$ & 18.9 & $2.00 /(1.80) \mathrm{L}$ & 0.73 & \\
\hline $\mathrm{k}$ & $517 / 518$ & $17 \mathrm{~S} / 23 \mathrm{~N}$ & 16.6 & $2.30 /(2.00) \mathrm{R}$ & $?$ & opt. ident.? \\
\hline $1+\mathrm{m}$ & $517 / 518$ & $24 \mathrm{~N} / 24 \mathrm{~N}$ & 16.6 & $2.30 /(2.00) \mathrm{R}$ & 0.69 & opt. ident.? \\
\hline $\mathrm{m}$ & 517 & $24 \mathrm{~N}$ & & $?$ & ? & ident.? \\
\hline $\mathbf{n}$ & 7 & $?$ & 16.6 & $2.30 /(2.00) \mathrm{R}$ & 0.79 & $\operatorname{limb}$ \\
\hline
\end{tabular}

\section{CONCLUSIONS}

The results have shown that the new spectral technique (PAS) used for solar observations with the radio telescope RATAN-600 allows unprecedented accuracy in the measurement of magnetic fields of sunspots compared with any method including optical. This 'radio' magnetic field is a new characteristic of a sunspot and the measured parameter refers to the highest value of the magnetic field in the region somewhere at the border of the corona and the chromosphere. The ratio of the radio to optical magnetic field is an essential parameter of a particular sunspot depending on both the distribution of magnetic field and temperature in the solar atmosphere above the spot. In this way one can expect to gain some information about the flux of energy in the magnetic tube of a sunspot. Further observations using this technique are necessary, especially those made with simultaneous optical measurements of magnetic fields at different levels of the photosphere and chromosphere.

\section{REFERENCES}

Abramov-Maximov, V.E. \& Gelfreikh, G.B. 1983, Pisma Astron. Zh., 9, 244

Akhmedov, Sh.B. et al. 1982, Solar Phys., 79, 41

Akhmedov, Sh.B. et al. 1983, Solar Phys., 88, 103

Bogod, V.M. \& Gelfreikh, G.B. 1980, Solar Phys., 67, 29

Bogod, V.M. et al. 1982, Solnechnye Dannye, No. 1, 104

Gelfreikh, G.B. 1972, Astron. Circ., No. 699, 3

Gelfreikh, G.B., Peterova, N.G., \& Ryabov, B.I. 1987, Solar Phys., 108, 89

Krüger, A., et al. 1986, Solar Phys., 105, 111

Zheleznyakov, V.V. 1970, Radio Emission of the Sun and Planets, (New York: Pergamon Press) 